\title{
Investigation of the maximum front to back load in the chairs made from curved and modified poplar wood
}

\author{
Ali Bayatkashkoli ${ }^{1}$ Mohsen Rahmani Ardakani ${ }^{1}$ Mahmoud-Reza Hosseini-Tabatabaei ${ }^{2}$. \\ Narjes Khaton Madahi ${ }^{1}$
}

Received: 17 November 2016/Accepted: 1 January 2017/Published online: 23 August 2017

(C) The Japan Wood Research Society 2017

\begin{abstract}
The aim of this research was to build a chair resistant to force from front to back. To achieve this goal, poplar wood was treated with heat and steam, and then curved at an angle of $90^{\circ}$. Curved frame was used for manufacturing chairs. In this study, three different designs of chairs were used. Control sample is a chair of custom type. The first design was a chair with a curved frame forming the structure back down to bottom (one-piece type) and the second design with two curved parts (twopiece type). Front to back load tests were conducted by mechanical universal testing machine in accordance with DIN EN 1729-2 standard. For each design, the test was repeated three times. The results of the experiment showed that differences between the designs were significant. The full type chairs were broken at the load about $1725 \mathrm{~N}$, but the partial type chairs at about $1421 \mathrm{~N}$. For the top to bottom load test, the full type chairs were broken at about $1052 \mathrm{~N}$, other chairs were more resistant. In general, by introducing a design using the curved frame made of treated poplar, the resistance of chairs is improved.
\end{abstract}

Keywords Wooden chairs - The maximum front to back load . The maximum top to down load Curved poplar wood

Ali Bayatkashkoli

ali.bayatkashkoli@gmail.com; ali.bayatkashkoli@uoz.ac.ir

1 Department of Paper, Wood Technology and Sciences, College of Natural Resources, University of Zabol, P.O. Box 98615-538, Zabol 9861673133, Iran

2 Department of Civil Engineering, College of Engineering, University of Zabol, Zabol, Iran

\section{Introduction}

Small-diameter poplar timber is not widely used in the furniture industry, because the morphological characteristics such as texture of wood, mechanical, and physical properties are not appropriate to woodworking. Various methods such as compression, coating, heat treatment, and chemical modification of wood are available, with which the poplar wood can be used in furniture industry.

On the other hand, fracture occurs in the joints of wooden structures. The joints are often reinforced with adhesive, stretcher, and shelf brackets. However, it is better to remove or reduce the number of joints. For this purpose, curved wood can be used instead to the jointed frame. In this study, chairs with curved frames were compared with conventional design. The aim of the study includes the use of reinforced frame and reduces the joints of frame for improving the quality of chairs.

Curved wood can be used in many ways, but deforming process of wood is difficult. Deforming of wood is easier when the wood is thin, so that the defects of curved wood can be prevented. In a uniform thickness such as chair legs, bent frame with two layers is of low cost and less equipment when compared with bent frame with multi-layers. Timber from small-diameter log such as poplar wood can be used for this process. Deforming and bending can be operated with various treatment methods. The treatment methods include the thermal treatment (hot water and steam), chemical materials, treatments through the combination of materials, pressure, and high temperature. Wood texture, its deformation characteristics, elastic or plastic behavior, and thermal treatment affect the efficacy of wood forming [1-3]. A lot of research have been presented in this area. 
Ou et al. [3] explored the thermoplastic deformation of poplar wood treated with ionic liquids. Ionic liquids can act as plasticizers to promote the thermal deformation of wood cell walls under pressure and high temperatures [3].

Liquid ammonia, chemical modifications such as esterification, benzylation, etherification, and also water with thermal flow, can act as a mild plasticizer for wood [4-7].

The laminated beams can be sufficiently deformed, but still keeps the elastic property. All the quantities of axial force, shear force, radial, and tangential displacements of laminated curved beam are expressed as functions of angle of tangent slope. The analytical solutions of laminated curved beams of circular and spiral were presented [8].

Deformation of curved laminated structural wood elements was studied [9]. The objective of the above study is to determine the effect of the wood type and the time on the deformations in a curved structural element manufactured with the lamination method. This analysis showed the wood type and time in the loading and unloading periods were not effective on the amount of deformation. There, the resulting 4-mm deformation is plastic deformation. Consequently, the amount of elastic deformation is $11 \mathrm{~mm}$.

Curved wood is a good idea to use the poplar wood to build a wooden chair. For manufacturing of chair with modified wood and curved parts, evaluation of the effect of curved components on the chair is necessary.

\section{Materials and methods}

Poplar trees (Populus nigra) without defects (slope of grain, knots, cracks, and split) were harvested from a plantation in Esfahan, Iran. Dimensional parts were cut from poplar trunks.

Polyvinyl acetate glue was used as an adhesive. The characteristics of the adhesive were as follows: the appearance color is milky white, smooth, and free of foreign particles; $\mathrm{pH} 3.2$; film-forming properties are smooth, colorless, and transparent; minimum film-forming temperature is $20^{\circ} \mathrm{C}$; storage time is 6 months; assembly time is $10 \mathrm{~min}$; preparation is not needed and the portion of solid material in the liquid adhesive is $32 \%$.

Seat frames were assembled with stainless screws of $40 \mathrm{~mm}$ length and diameter of $4 \mathrm{~mm}$.

\section{Preparation of wood for the manufacture of chairs}

Wood with high moisture content was cut from a tree. High moisture content of wood improves the curving process during manufacturing of chair. Wood element (fibers) at curved position gets fractured when moisture content of wood is low.
The sizes of dimension parts were $1800 \mathrm{~mm}$ length, $48 \mathrm{~mm}$ width, and $13 \mathrm{~mm}$ thickness. The chair frame was placed in a boiler tank for steaming. The thermal treatment (hot water and steam) was performed at a temperature of 130-125 ${ }^{\circ} \mathrm{C}$ for a period of $6 \mathrm{~h}$. The formatting pattern of frame was made from wood-based panels. The frame is curved in several parts. The types of molds are shown in Fig. 1.

Steamed dimensional parts were taken out from the chamber. Immediately, forming operation was performed. Each frame composed of two pieces overplayed. The frame on which the preliminary preparation was placed within a mold that was prepared in accordance with the desired form. Deformation operation was performed slowly. Frames were formatted by manual clamp and panel pieces. Chair frames resulted from this process. Wood fiber was crushed or compressed at curvatures and the location was supported with bolster component. Some defects are found at the curved points is often seen in bent wood. In this case, steel band other than the wood should be used in bending. Special form was pressed for a period of 7 days at a pressure of manual clamps (Fig. 2).

After the pressing operation, the frames were dried at kiln dry conditions for a period of 8-10 days. Curved dimensional parts must be tightened by rope. Drying defects of wood is prevented by fastener. After kiln drying, moisture content of wood was up to $6-8 \%$.

Two curved dimensional parts were glued together with face-to-face joints and polyvinyl acetate glue. Glue was spread uniformly on the dimensional parts manually and $160 \mathrm{~g} / \mathrm{m}^{2}$ adhesive was applied. The polyvinyl acetate glue was applied to the dimension parts by brush, and then the parts were assembled and clamped for $24 \mathrm{~h}$. Pattern of frame drying and assembled dimension parts are shown in Fig. 3. The specific molds were used for the deformation of chair frames. Three curved frames were formatted by the

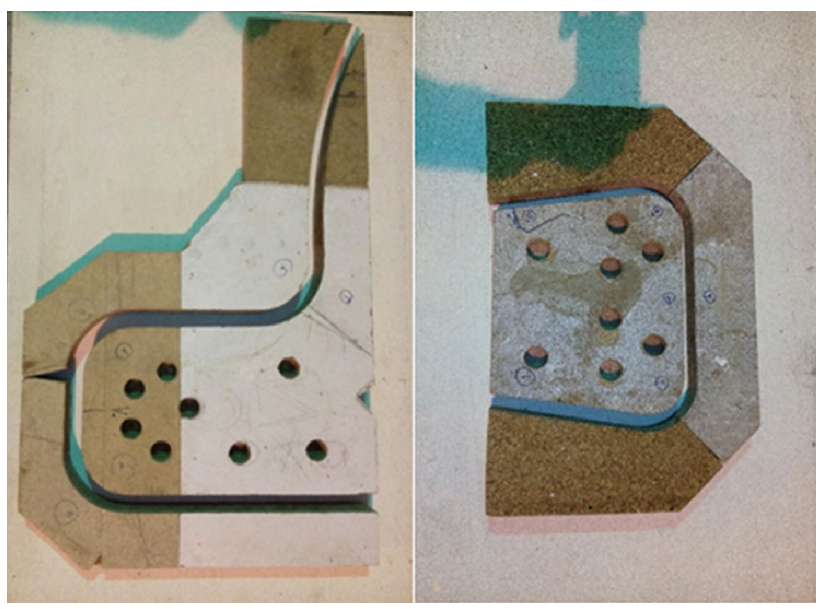

Fig. 1 Two specific molds for deforming of dimensional parts 


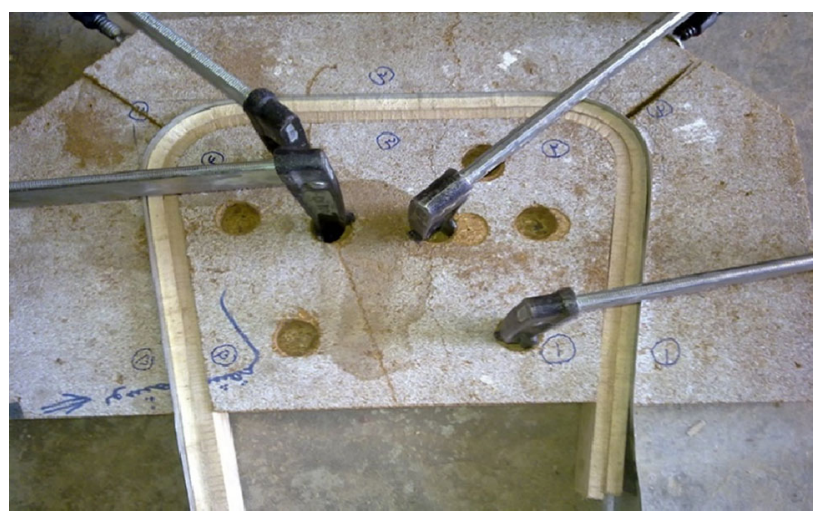

Fig. 2 Fastening clamps attached to the curved parts

specific molds. Fasten clamp has a key role at the deformation of chair frames. The defects such as check, crushed or compressed fibers, and fracture of bent wood can be resolved with the fasten clamp and bolster component. Each of the shaped frames was performed with simple tools and materials. Three-shaped frames are shown in Fig. 4. After the deformation operation as shown in Figs. 1 and 2, curved frames were fixed. The three-shaped frame was kept firmly as shown in Fig. 3. The curved frames were placed in a kiln and then dried in the same position.

Chair frames were clamped with fastener and clamp. The frames were kept at ordinal atmospheric conditions for a period of $24 \mathrm{~h}$, so that the adhesive used in the dimension parts become completely cured. The chair frames were finished by making cuttings at the edges and ends. Surface of curved frames were planed and sanded.

\section{The assembly of seats}

The final assembly of the chairs was accomplished with stainless screw in two different types of patterns (Figs. 4,
5). Another pattern is a custom-type chair as control sample. The structure of the custom chair is shown in Fig. 6. The dimensions of the chair and its components were decided based on a field survey and the DIN EN 1729-1 [10]. The designs for manufacture of the chair and their dimensions are shown in Figs. 4 and 6.

The one-piece type chair consists of long-span curved frames and the two-piece type consists of two short-span curved parts. The one-piece type and the two-piece type consist of two or three frames, respectively. The frame of the one-piece type is curved at three positions-bottom, front, and back (Fig. 4b). But the frame of the two-piece type was formatted at two locations (Fig. 4a). The curved parts were connected to each other by glue and stainless screws. In addition, horizontal support elements (medial stretchers) were used as connectors between the two curved elements. For each treatment, three replications were made.

\section{Experimental studies and test methods}

\section{Initial step}

Specimens in straight or curved forms manufactured from poplar wood were used. Three types of specimens were used. Two pieces glued together in curved or straight form were modified with thermal treatment, and another sample was untreated as control. These specimens were prepared for initial tests. All of the specimens were made of defect-free and straight grained poplar. Flexural properties of the specimens were determined according to the standards of ASTM D14394 [11]. All tests were performed on a computer-controlled INSTRON machine (Model DANP4). The
Fig. 3 Pattern of frames assembled with dimension parts in drying

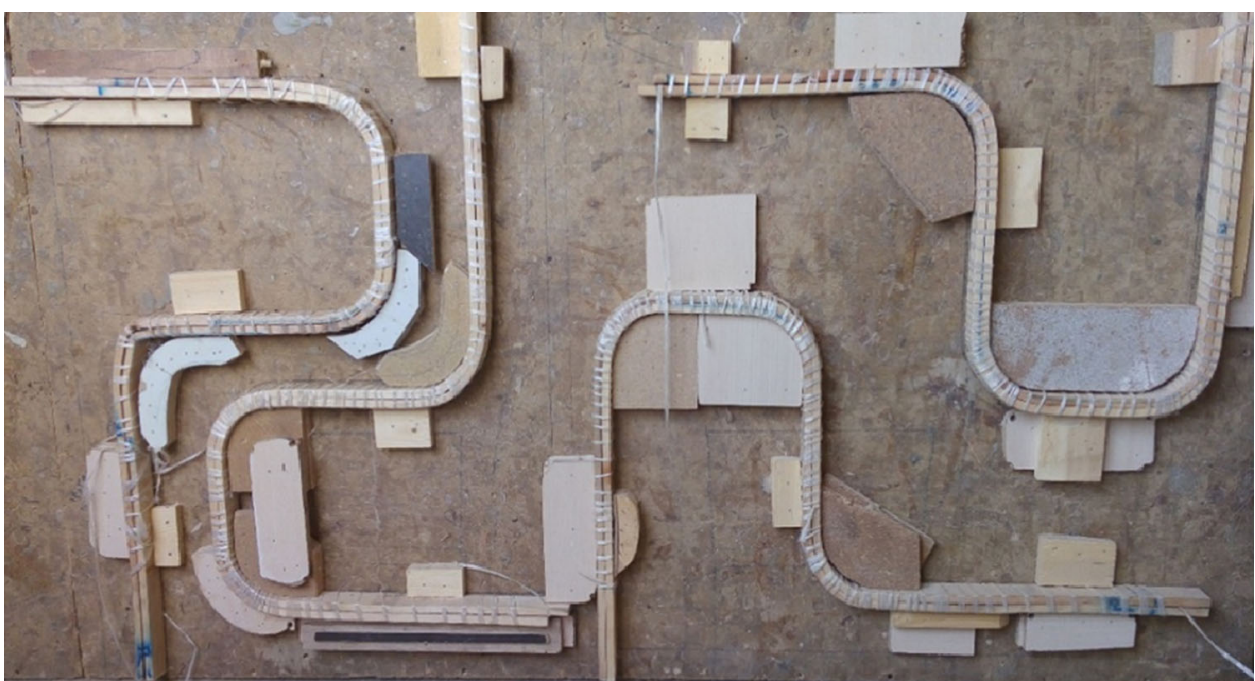


Fig. 4 Loading method applied from front to back on one-piece type chair (b) and two-piece type chair (a). a Two-piece type chair with three curved frames. b One-piece type chair with two curved frames. Arrow $F$ (purple arrows) is load direction
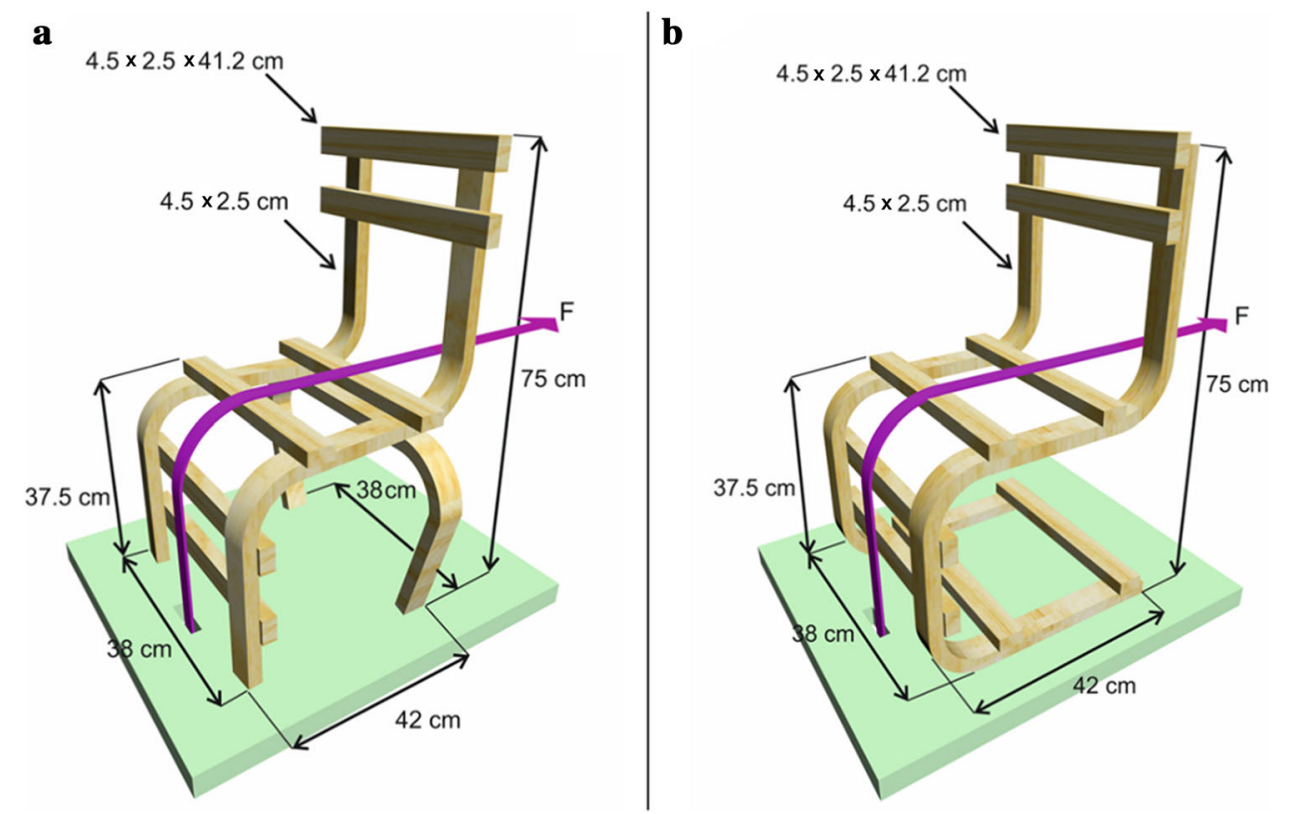

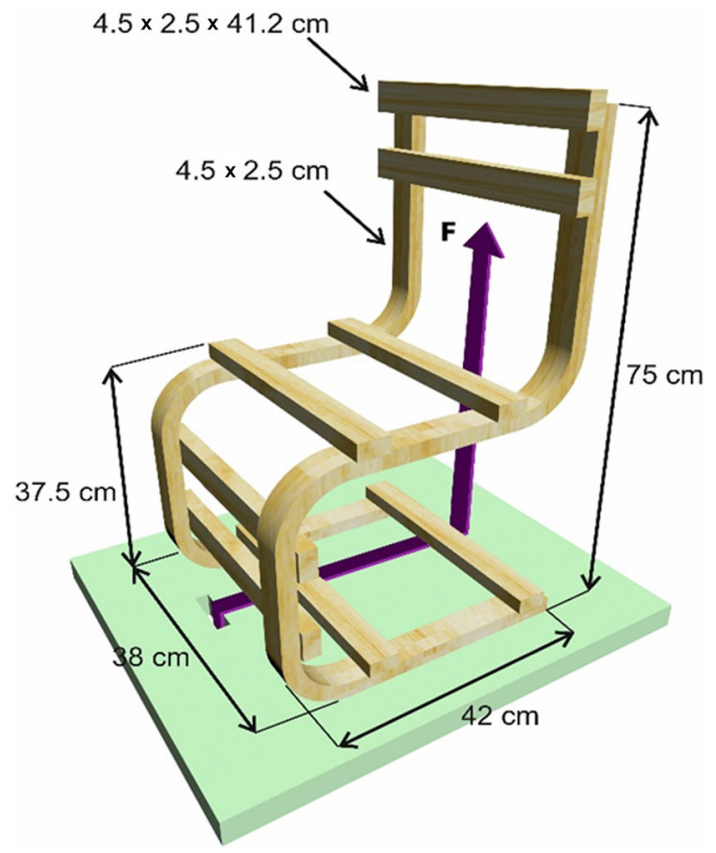

Fig. 5 Loading method applied from top to down on one-piece type chair. Arrow $F$ (purple arrows) is load direction

mechanical properties were measured based on bending load. In this study, bending and compression tests on the curved samples were performed. The test method is considered as a major factor in curved sample performance. This method of formulating the modulus of elasticity (MOE) and modulus of rupture (MOR) for curved samples was accomplished for the determination of curved sample behavior.

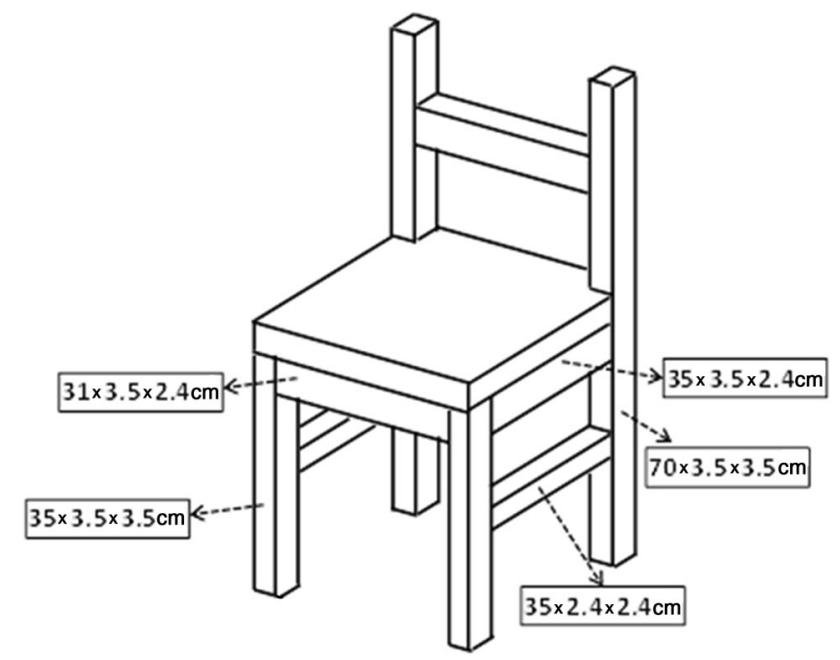

Fig. 6 The construction of manufacture of custom chair with parts' dimensions

The test methods of the samples are shown in Figs. 7 and 8. Loading was performed in compression or tension. The curved specimens were placed on the test station in accordance with the loading model given in Fig. 8.

Equations of MOE and MOR are calculated as follows.

The MOE and MOR of straight samples were calculated by the conventional formulas. There, the formula coefficients of MOE and MOR are 0.25 and 1.5, respectively, whereas the MOE and MOR of curved samples under bending loads (due to tension or compress loads) were calculated by other formulas. 

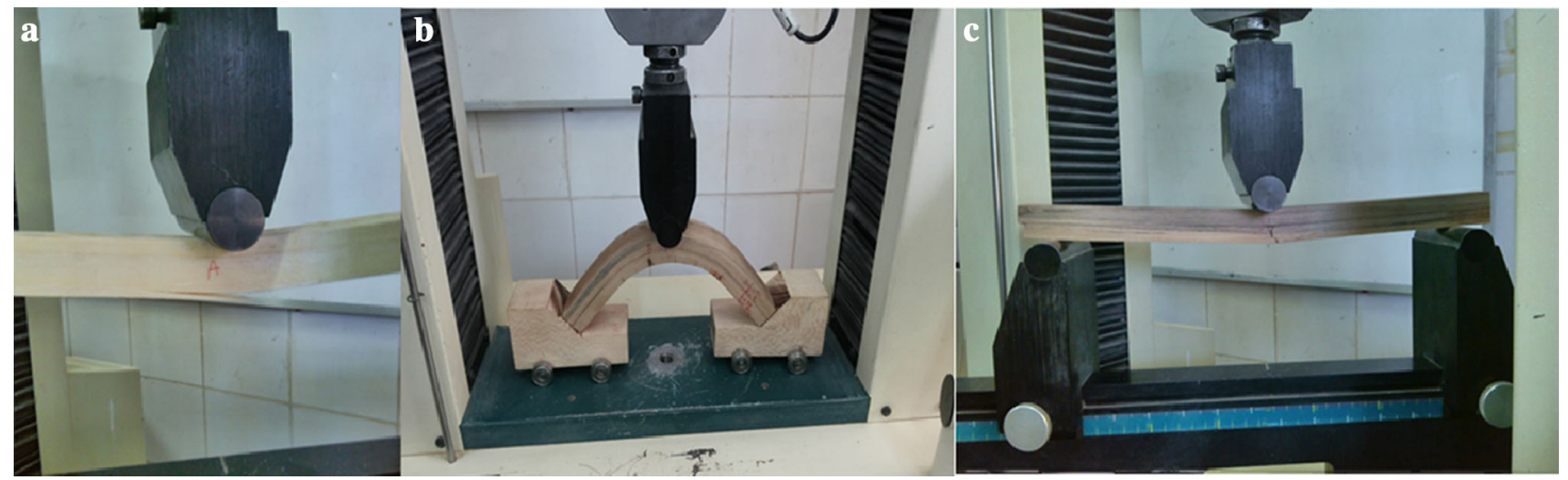

Fig. 7 Bending test on untreated control sample (a), two curved pieces glued together and modified with thermal treatment (b) and straight type (c)

Fig. 8 Bending and compression test on curved samples
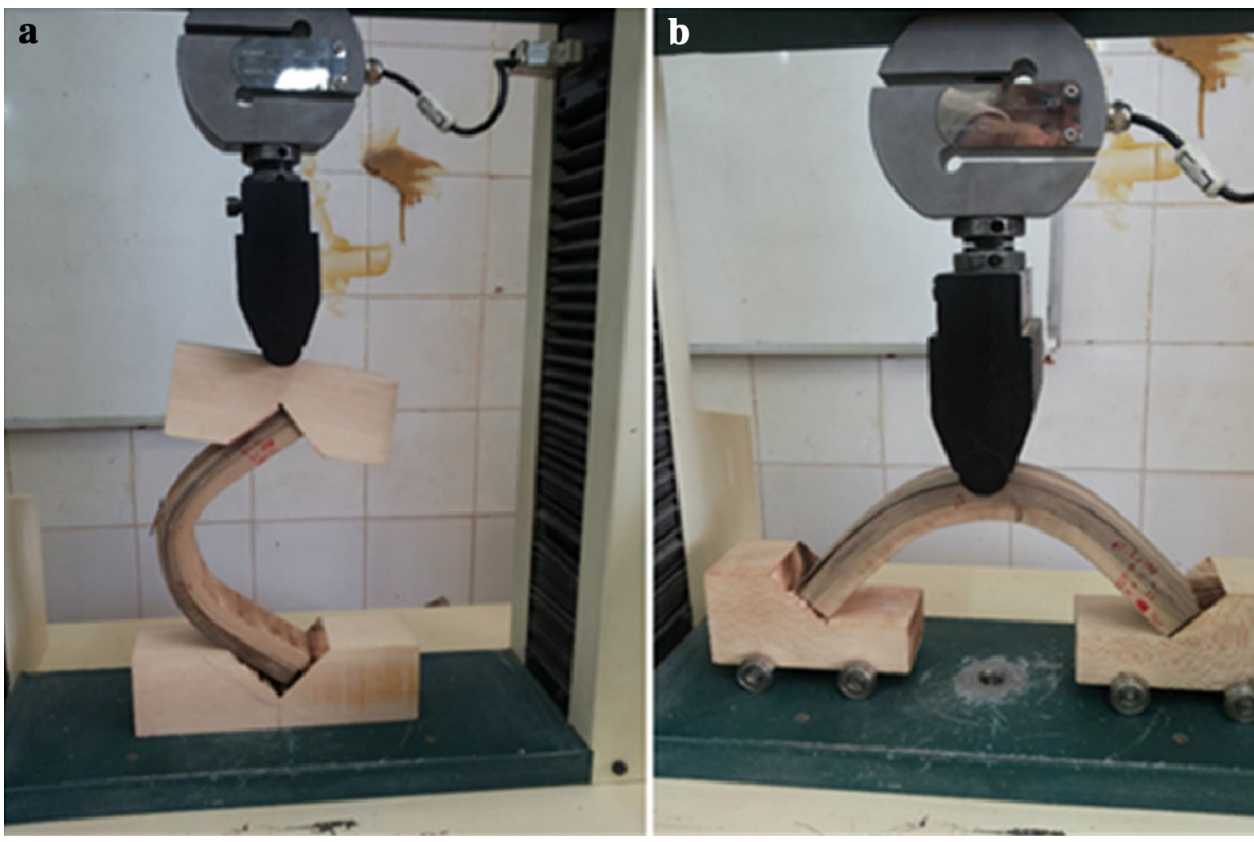

\section{Formulating the MOE and MOR for curved samples}

Here, it is assumed that the curvature radius is related to a full circle and formatting angle is $90^{\circ}$. Height of curved wood is equal to $85 \mathrm{~mm}$; width and thickness of cross section are 45 and $25 \mathrm{~mm}$, respectively. Curved samples were tested under tension or compression.

1. The process of formulations to achieve MOE and MOR for curved wood under tension loading is presented in the below lines (Fig. 9). Note: MOE is calculated using energy method. In addition, for the curved wood specimen determinate structures, MOR can readily be obtained applying equilibrium equations (Fig. 9).

2. The MOE and MOR formulations for curved wood under compression loading are shown in Fig. 10.

\section{Steps of performance test of chairs}

Front to back load tests on the chairs were conducted by mechanical universal testing machine in accordance with DIN EN 1729-2 standard [12]. Top to down load tests were performed on the one-piece type chair. The maximum loads were measured at the front to back load test or the top to down load tests.

A front to back load test was applied to each of the 12 chairs and also three chairs were prepared for top to down load tests.

The load was applied in a Universal Testing Machine. The loading method on the chair is shown in Figs. 4 and 5 . The chair was fixed so that it could not slip when applied the front to back load. The loading speed was about $6.5 \mathrm{~mm} / \mathrm{min}$ and the test was continued till a maximum load was reached [13-15]. 
Fig. 9 Curved wood under tensile loading on the inner surface

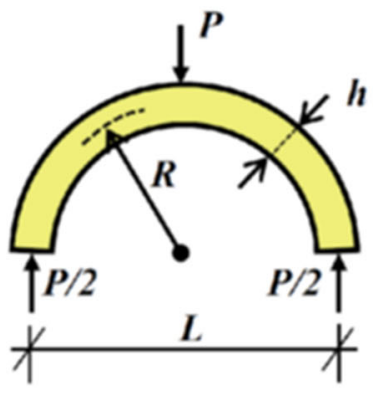

Three different designs of chairs were used. Three different designs of chairs include custom chair (Fig. 6), the one-piece type chair (Fig. 4b), and the two-piece type chair (Fig. 4a). In addition, two loading methods were applied that include front to back load (Fig. 4) and top to down load (Fig. 5). Three different designs and two loading methods are shown in Figs. 4 and 5.

\section{Analysis of the data}

The maximum loading test was designed according to a completely randomized design (CRD). The SPSS package program was used in the statistical analysis of the data. A one-way analysis of variance (ANOVA) was performed for the data. The differences between the means were investigated according to the least significant difference (LSD) test at a $95 \%$ level of protection.

\section{Results and discussion}

\section{Initial study}

The mean values of the MOE, MOR of control sample, modified sample with two straight pieces and modified sample with two curved pieces under tension load as shown in the LSD test are significantly different from each other.

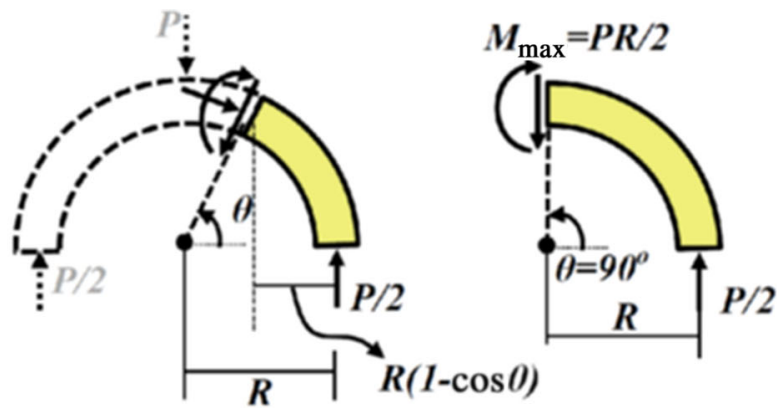

The means values of the MOE (along standard deviation) are equal to $2.86 \mathrm{E} 4( \pm 380), 2.66 \mathrm{E} 4( \pm 836)$, and $1.35 \mathrm{E} 3$ $( \pm 68.0) \mathrm{MPa}$ and also the means values of the MOR (along standard deviation) are equal to $71.2( \pm 1.8), 47.0( \pm 0.33)$, and $20.4( \pm 2.4) \mathrm{MPa}$, respectively. The mean values of the MOE and MOR of control sample, modified sample with two straight pieces and modified sample with two curved pieces under tension load are shown in Fig. 7. According to the analysis of variance, MOE and MOR are significantly influenced by bending wood at confidence level of $95 \%$ and also have a 0.999 and $0.995 R^{2}$. Loading diagram and failure position of initial samples are shown in Fig. 11.

The control sample has a high MOE and MOR. Therefore, the maximum load of control sample is higher than the modified wood. Note that this massive wood has a better performance than other samples. The decreasing strength of curved wood with two pieces is due to the thermal treatment of glued component parts when compared with samples of solid wood. LSD results shows MOE and MOR of specimens were significantly different from each other. The mechanical properties of curved woods (MOE, MOR, and maximum load) were reduced when the specimens were under tension load, but they were improved when the samples were under compression load. MOE, MOR, and maximum load of curved samples under compression load are equal to $1.23 \mathrm{E} 4,91.2$, and 5.03E3, which are more than curved samples under tension load.
Fig. 10 Curved wood under compressive loading on the inner surface

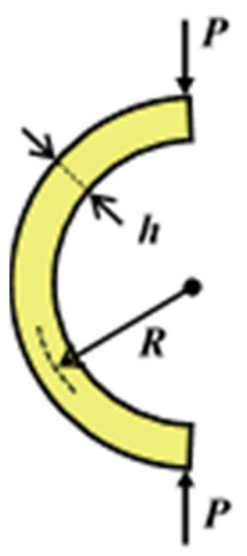

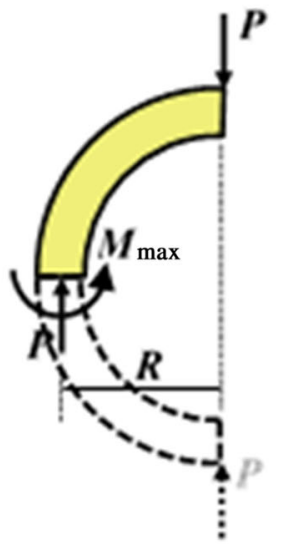

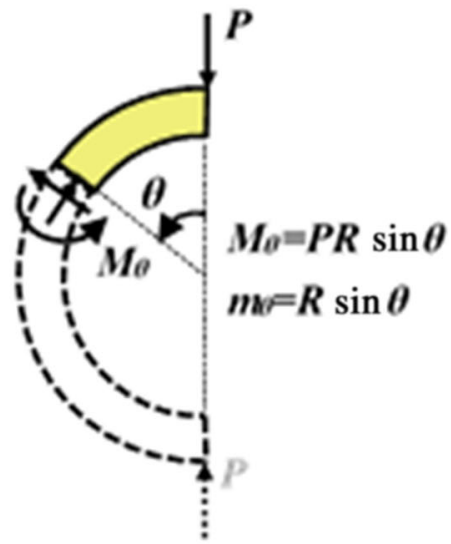


Fig. 11 Loading diagram and failure behaviors of samples in Fig. 7

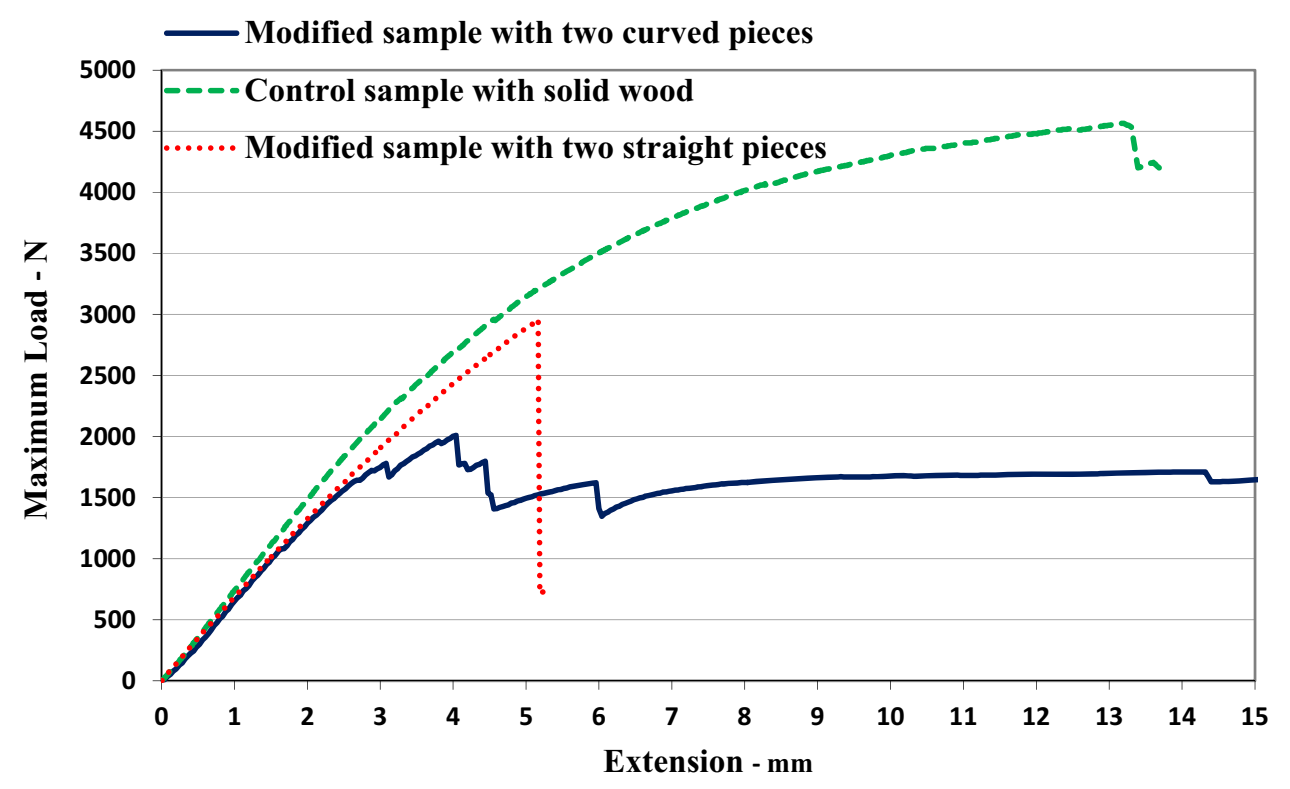

\section{Steps of performance test of chairs}

The mean values of the maximum load and standard deviation along with the LSD test for the front to back loads for control, one-piece type, two-piece type, and also one-piece type under the up to down load are $1.02 \mathrm{E} 3 \pm 38.9(\mathrm{C}), 1.73 \mathrm{E} 3 \pm 106(\mathrm{~A}), 1.42 \mathrm{E} 3 \pm 152(\mathrm{~B})$, and $1.05 \mathrm{E} 3 \pm 26.3(\mathrm{C}) \mathrm{MPa}$, respectively. The results of LSD show that the difference is significant at the 5\% level. Analysis of variance for treatments is shown in Table 1. One-piece type chair with two curved frames and twopiece type chair with three curved frames and U-shaped frame are shown in Fig. 4a, b. The front to back load type chairs are significantly different from each other, but the top to down load of the one-piece type chairs are is significantly different from the front to back load of the control samples. The front to back load of one-piece type chairs has more strength than two-piece type chairs, and then they resist more than custom chairs. The maximum load of one-piece type chair is reduced when the loading is top to down, but its maximum load is improved when the loading is under front to back. The top to down load of onepiece type chairs is similar to the front to back load of custom chairs and the difference between the samples is not significant. The custom chairs and two-piece type chairs have a high top to down load because of the chair legs (Fig. 6). One-piece type chairs with two curved frames are stronger than the rest under the front to back loading. Chairs of two-piece type or custom type were made from three frames or component parts glued together. The strength of chairs is decreased as the numbers of frames increased, for example, in the two-piece type chair and control samples.
Loading diagram and failure position of chair types are shown in Figs. 12 and 13. Failure locations at one-piece and two-piece type chairs are shown in Fig. 13. Failure occurred on the lower side of the frame of seat with entirely curved frame, but failure of two-piece type chair was located at the glue line between the back-curved frame and front-curved frames (Fig. 13). Compression and tension failure modes have occurred in the curved frame under compression and tension loads. Tension failure modes occur at the bottom of the curved frames of one-piece type chairs (b) and curved frame was fractured outside. In chair of type b, peeling occurs at the front-bottom of the curved parts. In chair of type a, peeling occurs at the connection point between seat-back and top of backside legs. In addition, a shear failure mode occurs at the curved frames of two-piece type chairs (a). Generally, the shear failure modes have been seen at both chair types. The top bent parts of frames are subjected to tension loads. The separations between two curved components of frames as shear modes have been seen at front-top. The difference between chair a (two-piece type) and b (one-piece type) is shown in Fig. 13. The custom chair joints that fail most frequently occur between the back post and the seat rail. When the frames were glued together, the front to back load of chairs is decreased. Failures usually occurred at the glue line. Chair of custom and two-piece type failed at the joints where the frames were glued together. Failure might have occurred in the curved location at the bottom such as in one-piece type chairs, since the failure of chair types was located at the formatted position or glue line. But chairs types with curved frames resisted the load. The curved frames have a better performance than the jointed frames. Curved frames resist the load from front to back. 
Table 1 Results of variance analysis of maximum loads related to chairs of various designs

\begin{tabular}{llrlll}
\hline Source & Type III sum of squares & $d f$ & Mean square & $F$ & Sig \\
\hline Corrected model & $1.006 \times 10^{6 \mathrm{a}}$ & 3 & $335,188.306$ & 36.850 & 0.000 \\
Intercept & $2.042 \times 10^{7}$ & 1 & $2.042 \times 10^{7}$ & $2.245 \times 10^{3}$ & 0.000 \\
Treatments & $1,005,564.917$ & 3 & $335,188.306$ & 36.850 & 0.000 \\
Error & $72,768.000$ & 8 & 9096.000 & & \\
Total & $2.150 \times 10^{7}$ & 12 & & & \\
Corrected total & $1,078,332.917$ & 11 & & & \\
\hline
\end{tabular}

$d f$ degree of freedom, $F F$ of statistical table calculated by mean squares (MS), Sig significant levels ${ }^{\text {a }} R^{2}=0.933$ (adjusted $R^{2}=0.907$ )

Fig. 12 Loading diagram and failure behaviors of chairs
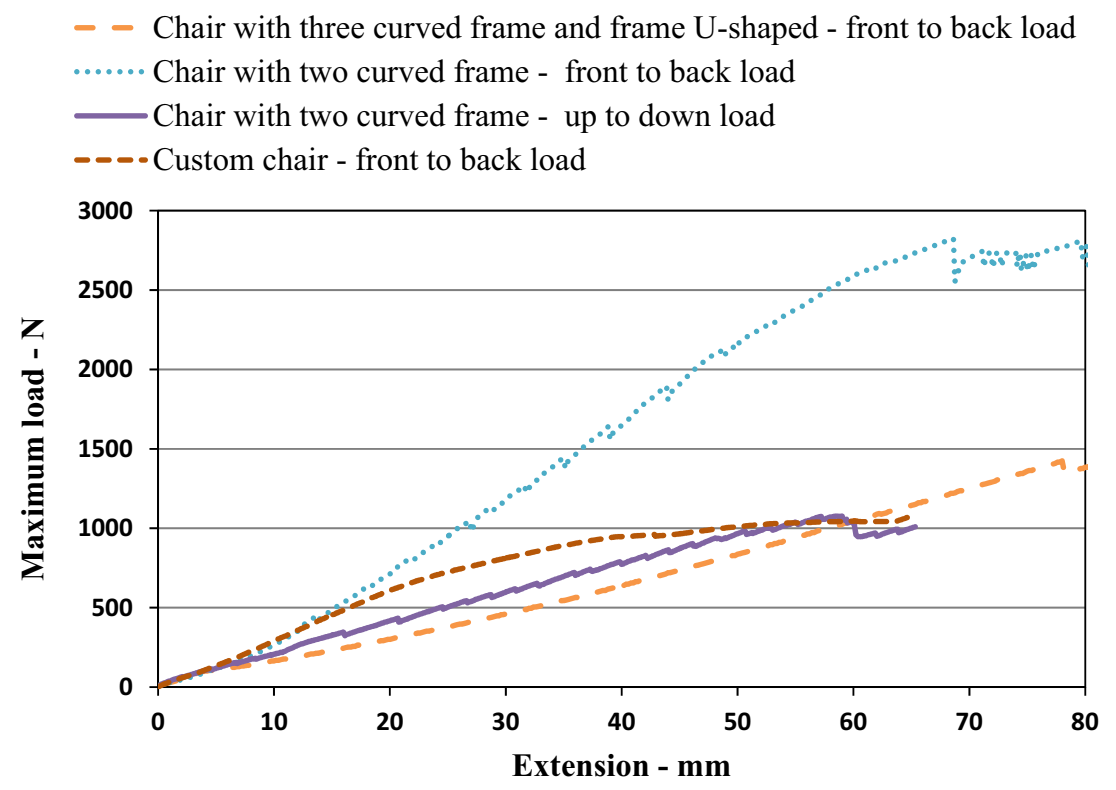

Performance of chairs can be clarified by the behavior of component parts. In addition, loading type is effective on the behavior of component parts. In this study, chairs are made from straight and curved parts. In practice, chairs with curved parts are under tension or compression load. Various failure modes of curved parts were different between tension load and compression load. Compression loads are applied to entirely curved frame of one-piece type (b) when the chairs are subjected to the top to down load or the front to back load. Tension failure locations of curved frame have occurred in the bottom. But shear failure modes appeared in curved frame of two-piece type (a) when the chairs are subjected to the front to back load. Two curved dimensional parts were separated under tension load. The shear failure locations of curved frame were found in the front-curved legs. The various failure modes of curved parts are shown in Fig. 13. Since performance of chairs is similar to curved samples, and these properties are known in the initial step of the study and step of performance test of chairs, the first step in this study was performed to identify the behavior of component parts in the chairs.
In this study, it was aimed to increase the strength of the weakest part that is located at the joint between the back post and side rail. The weak part affected the front to back load of chairs. The front to back load depends on the chair frames. The component parts with joint types are less strong than without joint. Stretcher types (box stretcher and cross stretcher) and wood shelf brackets were used to reinforce chair frames.

Stretcher type and brackets changed the failure location at the joint between the back or front post and side rail. In this study, strengthening the frames of chairs includes stretcher and bracket used in the control samples. The maximum front to back load was obtained with the curved frames of chairs when compared to control samples. Onepiece and two-piece type chairs have an important advantage in comparison with control samples, if only the front to back load is noted. The comparisons with custom chairs very clearly show the superior performance of the curved frames of one-piece type and two-piece type chairs. The high load at front to back was obtained with the curved frames, especially for one-piece type chairs. The front to 


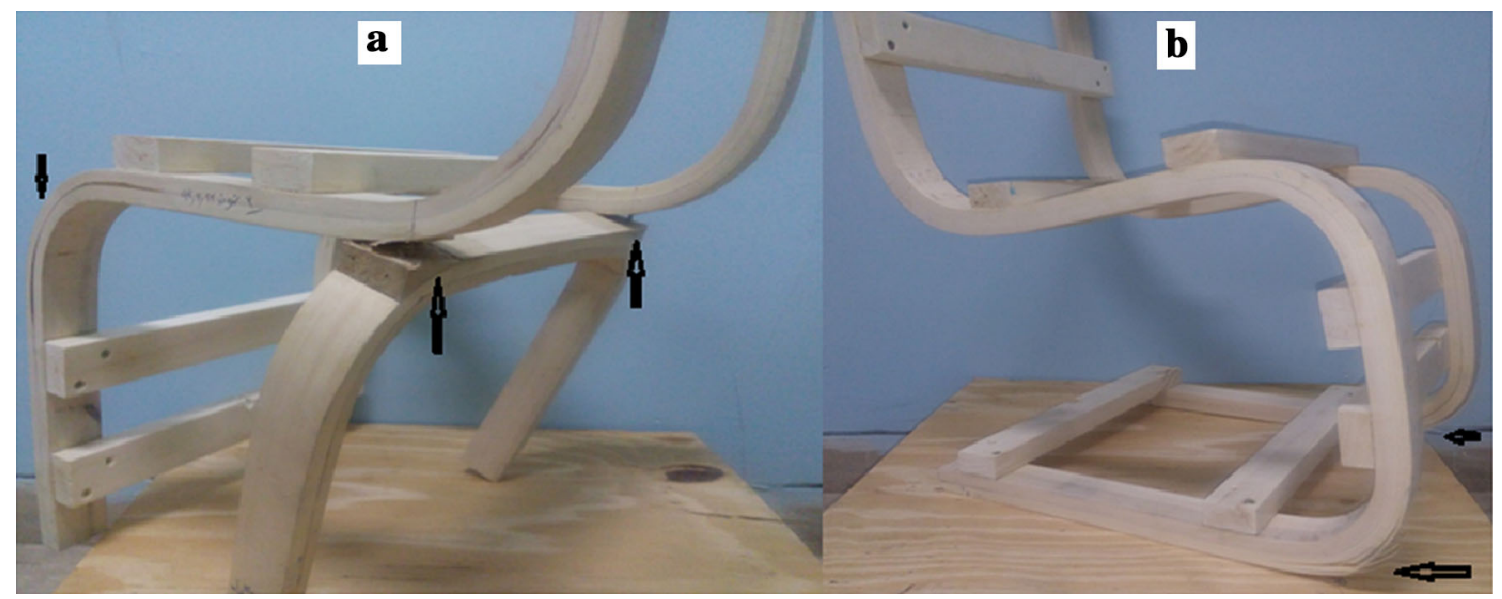

Fig. 13 Failure locations that occurred at curved frame or glue line. Shear failure and separation of glue line in two-piece type chair (a). Tension failure in one-piece type chair $(\mathbf{b})$

back loads of first, two-piece type chairs, and custom chairs are equal to $1.72 \mathrm{E} 3,1.42 \mathrm{E} 3$, and $1.02 \mathrm{E} 3 \mathrm{~N}$, respectively. The increasing strength of chairs with curved frames is due to the removal of joints when compared with chairs of solid wood.

The DIN EN 1729-2 [12] is a safety standard required for test methods related to chairs and tables for educational institutions. The strength and stability of chairs are determined by the test method. Various types of loading test were applied to the chairs. Loading tests include the front to back load, the back to front load, the top to down load and side thrust load. In practice, the front to back load is important for custom chair. The joint between the back post and side rail of the custom chair is the weak point against the applied loads. The weakest chair part can be reinforced with curved frames. But the stability of seats depends on the chair frames. For example, the chair with curved frames is weak against top to down load.

The formula coefficients of MOE and MOR are 0.25 and 1.5 that are related to straight pieces. According to formulation, the MOE and MOR for curved sample under tension or compression loads have more estimated quantity than value amount of the control samples, whereas the MOE and MOR of curved sample are decreased for applied load through the formatting processes. The front to back loads of one-piece and two-piece type chairs as shown in Fig. 12 are improved. The increasing strength of chairs with curved frames is due to their design and less number of joints when compared with custom chairs. Note that this chair has a better performance than custom chair where the loading is applied under front to back. The one-piece type chairs (B) are less strong than other chairs when the loading is applied under top to down. The legs of chairs have a key role against top to down load. The bending properties of curved samples are decreased for the combined effect such as pressure and high temperature. But the strength and stability of structures like a chair with curved frames are more than the custom chairs. The effect of curved components on the chair is shown in the loading diagram (Fig. 12). The stiffness and strength of chair B with two curved frames (one-piece type chair) as shown in Fig. 12 have improved when compared to the loading diagram of the custom chair.

Initially, improving the resistance of chairs with curved and modified poplar wood was unclear. The front to back load tests on the chairs showed that the front to back load is improved in this study. But the top to down load of chairs made with entirely curved frame (one-piece type chair) has been reduced. As a primary solution, a chair like in Fig. 14 with more parts is strong. Drawn from top to bottom of one-piece type chairs is $1052 \mathrm{~N}$ and hereof, other chairs are more resistant than the one-piece type. This low load of top to down in one-piece type chairs can be removed by a vertically curved stretcher as shown in Fig. 14.

Samples of solid wood are stronger than modified and curved samples. However, due to the removal of the joints of frames, chair structures with curved frames become stronger than the custom chairs with solid wood. Surely, the curved frame has more strength than the jointed frame.

The anatomical structure of poplar wood weakens the wood against applied stress. A large deformation took place in the area of earlywood vessels, rays, and libriform fibers [3]. Decrease in the strength of modified woods was apparent. However, if loading distribution is appropriated at curved frames of chairs, this frame has excellent performance. Performance of one-piece type chair can be further improved by various curved stretchers.

The curved beam is such that the dimension of the cross section is much less than the dimension of the radius of curvature [8]. In this study, deformation operation was 


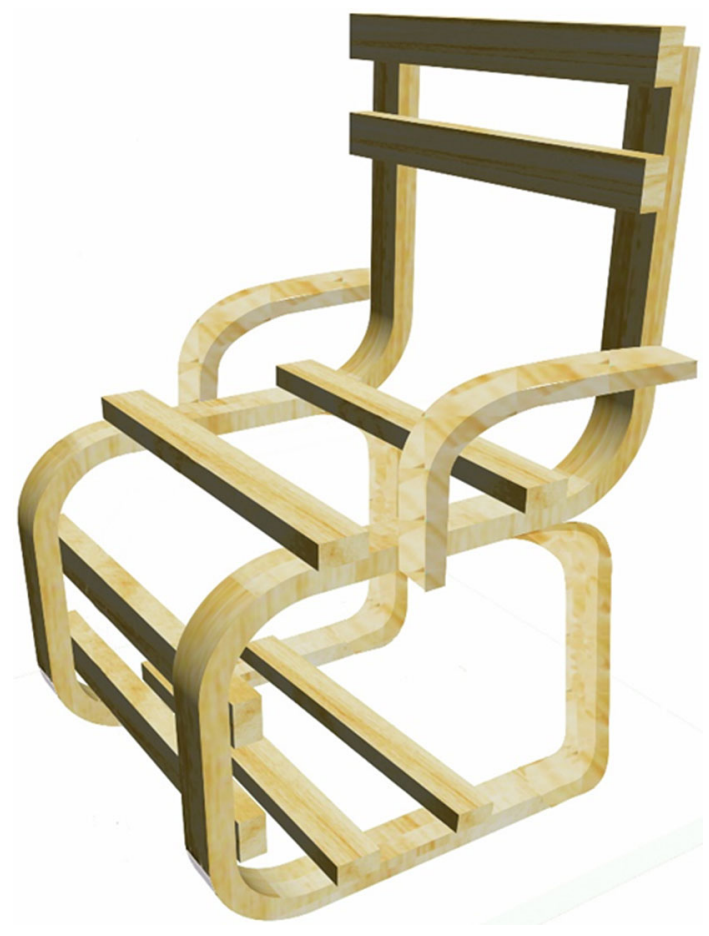

Fig. 14 Suggested improved design of higher strength chair

performed easily, because the thickness of curved wood was thin.

The MOE and MOR of curved samples under tension loading are estimated to be three and two times larger than the control samples and also MOE and MOR of curved samples under compression loading are six and four times larger than the control samples, respectively. But bending strength of curved wood is decreased due to cell wall fracture and deformation of wood cells by the applied compression stress through the combined effect of pressure and high temperature. The failure modes of thermal treatment sample are brittle, and the failure modes of curved wood show the mobility and flexibility, when compared to control samples (Fig. 11). The formatting operation can be adjusted according to the failure modes. Finally, the usage type of curved wood has a great role for an excellent performance of chair structures. In addition, modified wood has more advantages such as dimensional stability when compared to solid wood.

Deformation of curved laminated structural wood elements was analyzed by Altinok et al. [9]. The modified poplar woods were put within a mold that was prepared in accordance with the desired form and were pressed for a period of $120 \mathrm{~min}$ at a pressure of $2.5 \mathrm{~N} / \mathrm{mm}^{2}$. After the pressing operation, the specimens were kept at ordinal atmospheric conditions for a period of 3 weeks for the adhesive used in the lamination to become completely cured [9]. Poplar woods of green and sound are preferred for easy deforming. The poplar woods were subjected to a formatting process in a handled deformation mold until it reached a radius curvature of $90^{\circ}$. Deforming operation is performed gradually and the specimens were kept at ordinal atmospheric conditions for a period of 1 week, for their deformation in the frames to become completely fixed. In this situation, clamped frames were dried in a drying kiln until it reached a moisture content of $10 \%$. Therefore, they retained their new positions and the deformation was not recoverable. In addition, warp and check defect of frames can be eliminated in the processes.

Curved samples under compression load have a superior performance when compared to the samples subjected to tension load. In practice, entirely curved frame of onepiece type is subjected to compression load. The strengths of the frames of the one-piece type chairs are due to compressing load because the top to down load on the onepiece type chairs are performed as compressing load. As it is known, the top to down load on the one-piece type chairs is similar to the front to back load on the control samples. The strength of chair structures is obtained with the curved frames and the maximum load for various failure modes is different from custom chairs with solid wood. Therefore, deformation in wood is one of the important factors affecting the strength of chair frames. By comparing the maximum load of custom chair and other chairs, it was found that the front to back loads of chair on curved frames are estimated 1.5 times. The superior performance of chair with curved frames is due to the design type of the chair. The chair with new design type can also be suggested as shown in Fig. 14. Structural form and the radius of the curve are directly influential on the resistance characteristics and the amount of deflection [9]. Curved stretchers as shown in Fig. 14 can improve the strength of chairs. Suggested design of chair can improve the maximum load, especially for the top to down load.

\section{Conclusion}

The removal of the joints of frame is one of the methods for the frame reinforcement. Instead of the jointed frame, curved wood can be used. For instance, in chair design, the chair can be manufactured using curved frame. The curved wood is one of the important factors affecting the strength of chair frame, and the curved frames are directly influential on the maximum load of chair. Another advantage is realized by use of modified poplar wood. Poplar wood is of low quality for furniture structures, but the thermal treatment (hot water and steam) and formatting of wood increase the efficacy of the poplar wood. Optimization of the distributions of loads is an important factor for curved frames compared with control samples, and the frame without joints has an excellent performance. Wooden 
chairs made with two types of curved frames are stronger than the custom chairs. One-piece type chairs with two entirely curved frames is stronger than two-piece type chairs with three curved frames, when front to back loading was applied. But the strength improved for the top to down load on two-piece type chairs. As a proposal, the chair with vertical stretcher can be better against the loads, especially for top to down load. Failures usually occurred at the glue line or formatted position. First, chair failed at the joints where the frames were glued together and then failure might have occurred in the formatted position of chair frames. The curved location at the bottom such as frames of one-piece type chairs and glue line at other chairs disposed to failure. In final, curved frame at the maximum load of chair is considered and the highest strength is obtained by formatted wood without joints.

\section{References}

1. Yamaguchi K, Otsubo M, Motomura Y, Marumo Y, Shiozaki Y, Kawase T (2006) Deformation characteristics of wood structures under compression. Mater Sci Forum 505:1051-1056

2. Saje M, Turk G, Kalagasidu A, Vratanar BA (1998) Kinematically exact finite element formulation of elastic-plastic curved beams. Comput Struct 67:197-214

3. Ou R, Xie Y, Wang Q, Sui S, Wolcott MP (2014) Thermoplastic deformation of poplar wood plasticized by ionic liquids measured by a nonisothermal compression technique. Holzforschung 68:555-566
4. Pentoney RE (1966) Liquid ammonia-solvent combinations in wood plasticization. Properties of treated wood. Ind Eng Chem Prod Res Dev 5:105-110

5. Schuerch C, Burdick MP, Mahdalik M (1966) Liquid ammonia solvent combinations in wood plasticization. Chemical treatments. Ind Eng Chem Prod Res Dev 5:101-105

6. Shiraishi N (2000) Wood plasticization. In: Hon DNS, Shiraishi N (eds) Wood cellulosic chemistry. Marcel Dekker Inc, New York, pp 655-700

7. Kelley SS, Rials TG, Glasser WG (1987) Relaxation behaviour of the amorphous components of wood. J Mater Sci 22:617-624

8. Lin KC, Lin CW (2011) Finite deformation of 2-D laminated curved beams with variable curvatures. Int J Non-Linear Mech 46:1293-1304

9. Altınok M, Burdurlu E, Ozkaya K (2008) Deformation analysis of curved laminated structural wood elements. Constr Build Mater 22:1643-1647

10. DIN EN 1729-1 (2006) Furniture-chairs and tables for educational institutions. Part 1: Functional dimensions. Deutsches Institut für Normuug (DIN), German

11. ASTM (2005) Standard methods of testing small clear specimens of timber. American Society for Testing and Materials (ASTM), West Conshohocken

12. DIN EN 1729-2 (2006) Furniture-chairs and tables for educational institutions. Part 2: Safety requirements and test methods. Deutsches Institut für Normuug (DIN)

13. Eckelman C (1999) Performance testing of side chairs. Eur J Wood Wood Prod 57:227-234

14. Eckelman C, Haviarova E (2006) Performance test of school chairs constructed with round mortise and tenon joints. For Prod J $56: 51-57$

15. Haviarova E, Eckelman C, Erdil Y (2001) Design and testing of environmentally friendly wood school chairs for developing countries. For Prod J 51:58-64 